\title{
Precessional Constant Determined from Optical Astrometry
}

\section{Miyamoto}

National Astronomical Observatory, Mitaka, Tokyo 181, Japan

We have analyzed proper motions of about $30000 \mathrm{~K}-\mathrm{M}$ giants chosen from the astrometric catalogue ACRS Part 1 (Corbin and Urban 1991). To avoid localized velocity fields, these K-M giants are chosen from the heliocentric distance interval $0.5-1.0 \mathrm{kpc}$. Starting from the initial trial of the luni-solar precessional correction $\Delta p \sim-0^{\prime \prime} .3 / c y$, we have found that the motion of stars are well expressed in terms of the Oort constants for the K-M giants. After confirming that the K-M giants are in a steady-state, we have applied the velocity-field model of the plane-parallel galactic rotation with non-zero Oort constants. Then, we have found a rational set of the corrections to the FK5 system: $\Delta p=-0^{\prime \prime} .27 \pm 0^{\prime \prime} .03 / \mathrm{cy}$ and $\Delta e+\Delta \lambda=-0^{\prime \prime} .12 \pm 0^{\prime \prime} .03 / \mathrm{cy}$, as is shown in Table 1 (Miyamoto and Sôma 1993). In consequence, the FK5 system is still rotating.

TABLE I

Kinematic Parameters Derived from Proper Motions Given by ACRS Part 1

\begin{tabular}{|c|c|c|c|}
\hline $\begin{array}{l}\text { Kinematic } \\
\text { Parameters }\end{array}$ & $\begin{array}{c}\text { Least Squares } \\
\text { Method } \\
\text { K-M Giants } \\
|z| \leq 0.5 \mathrm{kpc}\end{array}$ & $\begin{array}{c}\text { Maximum Likelihood } \\
\text { Method } \\
\text { K-M Giants } \\
|z|<1.0 \mathrm{kpc}\end{array}$ & $\begin{array}{c}\text { Least Squares } \\
\text { Method } \\
\text { Young Stars } \\
0.5 \mathrm{kpc} \leq r \leq 3.0 \mathrm{kpc}\end{array}$ \\
\hline$\Delta p(" / \mathrm{cy})$ & $-0.267 \pm 0.028$ & $-0.214 \pm 0.022$ & -0.27 (given) \\
\hline$\Delta e+\Delta \lambda(" / c y)$ & $-0.116 \pm 0.026$ & $-0.075 \pm 0.037$ & -0.12 (given) \\
\hline$S_{1}(\mathrm{~km} / \mathrm{s})$ & $+13.6 \pm 0.3$ & $+13.4 \pm 0.31$ & $+8.7 \pm 0.8$ \\
\hline$S_{2}(\mathrm{~km} / \mathrm{s})$ & $+23.3 \pm 0.3$ & $+20.3 \pm 0.38$ & $+15.9 \pm 0.8$ \\
\hline$S_{3}(\mathrm{~km} / \mathrm{s})$ & $+11.9 \pm 0.3$ & $+12.2 \pm 0.22$ & $+9.1 \pm 0.7$ \\
\hline$S_{\text {total }}(\mathrm{km} / \mathrm{s})$ & 29.5 & 26.7 & 20.3 \\
\hline$A=D_{12}^{+}(" 1 /$ cy) & $+0.263 \pm 0.012$ & $+0.243 \pm 0.011$ & $+0.285 \pm 0.019$ \\
\hline$B=D_{21}^{-}\left({ }^{\prime \prime} / \mathrm{cy}\right)$ & $-0.176 \pm 0.010$ & $-0.193 \pm 0.010$ & $-0.260 \pm 0.015$ \\
\hline$V_{\theta}(\mathrm{km} / \mathrm{s})$ & $-177.1 \pm 6.2$ & $-175.7 \pm 6.0$ & $-219.9 \pm 9.8$ \\
\hline$D_{13}^{+}$ & 0 & 0 & $-0.059 \pm 0.011$ \\
\hline$D_{13}^{-}$ & 0 & 0 & $+0.059 \pm 0.011$ \\
\hline$D_{23}^{+}$ & 0 & 0 & $+0.039 \pm 0.010$ \\
\hline$D_{32}^{-}$ & 0 & 0 & $+0.039 \pm 0.010$ \\
\hline$D_{z z}\left(\mathrm{~km} / \mathrm{s} / \mathrm{kpc}^{2}\right)$ & - & $15.6 \pm 2.2$ & - \\
\hline$\sigma_{R}(\mathrm{~km} / \mathrm{s})$ & - & $+31.3 \pm 0.4$ & - \\
\hline$\sigma_{\theta}(\mathrm{km} / \mathrm{s})$ & - & $+25.2 \pm 0.5$ & - \\
\hline$\sigma_{z}(\mathrm{~km} / \mathrm{s})$ & - & $+21.2 \pm 0.5$ & - \\
\hline$\epsilon_{\mu a}(" / \mathrm{cy})$ & - & $0.56 \pm 0.02$ & - \\
\hline$\epsilon_{\mu \delta}(" 1 / c y)$ & - & $0.52 \pm 0.02$ & - \\
\hline $\begin{array}{l}\text { total number } \\
\text { adopted }\end{array}$ & 20292 & 22629 & 1892 \\
\hline
\end{tabular}

\section{References}

Corbin, T.E. and Urban, S.E. (1991) Astrographic Catalogue Reference Stars (ACRS), U.S. Naval Observatory. Miyamoto, M. and Sôma, M. (1993) AJ, 105, 691. L9. 